\title{
ANOTHER PROOF OF THE LIOUVILLE THEOREM
}

\author{
Zhuomin Liu \\ University of Pittsburgh, Department of Mathematics \\ 301 Thackeray Hall, Pittsburgh, PA 15260, U.S.A.; zhl26@pitt.edu
}

\begin{abstract}
We provide another proof of the Liouville theorem that conformal mappings in the dimensions at least three are Möbius transformations under the assumption that the mapping is 1-quasiconformal. Our method employs the Ahlfors Cauchy-Riemann operator.
\end{abstract}

\section{Introduction}

The celebrated Liouville theorem from 1850 [17], states that the only conformal mappings in a domain $\Omega \subset \mathbf{R}^{n}$, where $n \geq 3$, are restrictions of Möbius transformations to $\Omega$. The situation here is much more rigid than in dimension two, where we have plenty of conformal mappings. Liouville's proof required the mapping to be a diffeomorphism of class at least $C^{3}$, and many subsequent proofs also required that regularity. It is worth to mention here the proof by Capelli [5, 24], and the most commonly known proof by Nevanlinna [20, 7]. Actually Nevanlinna's proof requires the mapping to be of class $C^{4}$.

On the other hand $C^{1}$ regularity is sufficient to define conformal mappings and one may inquire whether Liouville's theorem remains true under that condition. The reduction of assumptions from $C^{3}$ to lower regularity turned out to be very difficult. Hartman [11, 12], proved the Liouville's theorem for $C^{2}$ mappings in 1947 and for $C^{1}$ mappings in 1958.

With applications to the theory of quasiconformal mappings and nonlinear elasticity one needs to consider conformal mappings under still weaker assumptions. Subsequently Gehring [8] in 1962 proved the theorem for 1-quasiconformal mappings and Reshetnyak [21] in 1967 for 1-quasiregular mappings. Both approaches were based on deep regularity results for the solutions to the nonlinear $n$-harmonic equation $\operatorname{div}\left(|D u(x)|^{n-2} D u(x)\right)=0$. Note that 1-quasiconformal or more generally 1-quasiregular mappings are in the Sobolev space $W_{\text {loc }}^{1, n}(\Omega)$. An elementary, but rather involved proof of Reshetnyak's result [21] was given by Bojarski and Iwaniec [4] in 1982, see also [14]. Further developments have arisen from the work Iwaniec and Martin [15], where they further reduced the assumption of $f \in W_{\text {loc }}^{1, n}$ to $f \in W_{\text {loc }}^{1, \frac{n}{2}}$ weakly 1-quasiregular mappings in even dimensions. On the other hand in any dimension $n \geq 3$ there are known examples [15] of weakly 1-quasiregular mappings in $f \in W_{\text {loc }}^{1, p}$ for $p<\frac{n}{2}$ that are not Möbius transformations. The question whether $f \in W_{\text {loc }}^{1, \frac{n}{2}}$ weakly 1-quasiregular mappings are Möbius transformations in odd dimensions remains a long standing open problem. Furthermore, Iwaniec [13] also proved that in all dimensions we can relax the assumption to $W^{1, n-\epsilon}$ for some $\epsilon>0$.

doi:10.5186/aasfm.2013.3807

2010 Mathematics Subject Classification: Primary 35B53, 30C35, 30C65; Secondary 46E35.

Key words: The Liouville theorem, 1-quasiconformal mappings. 
Recently, Liu [18] proved the Liouville theorem for $W_{\text {loc }}^{1,2 p} 1$-quasiregular mappings under one additional assumption $|D f|^{p} \in W_{\text {loc }}^{1,2}$ for $p \geq(n-2) / 4$. This paper also suggests that the Iwaniec-Martin conjecture can be reduced to new a conjecture about a Caccioppoli type estimate that reflects the importance of $n / 2$.

One more proof worth mentioning is the one given by Sarvas [23], under the $C^{2}$ regularity assumption: For a mapping $f \in C^{1}$, Ahlfors [1], introduced a linear Cauchy-Riemann operator

$$
S f=\frac{1}{2}\left(D f+D^{T} f\right)-\left(\frac{1}{n} \operatorname{div} f\right) \cdot \mathrm{I} .
$$

The mapping $f$ is called a trivial deformation if $S f=0$. Ahlfors proved that a trivial deformation is a polynomial of degree 2 and Sarvas showed that if $f \in C^{2}$ is a conformal diffeomorphism, then for any $b \in \mathbf{R}^{n},[D f(x)]^{-1} b \in C^{1}$ is a trivial deformation and Liouville's theorem follows from this result.

The purpose of this paper is to provide a different proof of the Liouville theorem for 1-quasiconformal mappings using the Ahlfors operator. I believe that this proof is more geometric and hence more natural than the previous proofs. One of the motivations to use the Ahlfors operator was a statement by Iwaniec and Martin [15, p. 37]: However as first degree (linear) approximations of the nonlinear system of equations for conformal mappings, the Ahlfors operators are rather difficult to use.

\section{Notations and the main theorems}

If $f: \Omega \rightarrow \mathbf{R}^{n}, \Omega \subset \mathbf{R}^{n}$, where $\Omega$ is a domain in $\mathbf{R}^{n}$, is a diffeomorphism of class $C^{1}$, it is easy to see that it is conformal if and only if $D f^{T}(x) D f(x)=|J f(x)|^{\frac{2}{n}} \cdot \mathrm{I}$ for all $x \in \Omega$. Here $J f(x)$ is the Jacobian of the mapping $f$ and I is the identity matrix.

Recall that the Sobolev space $W^{1, p}$ consists of functions in $L^{p}$ whose distributional derivatives are also in $L^{p}$. Similarly we define $W_{\text {loc }}^{1, p}$. The notion of conformal mappings can be generalized to the Sobolev settings as follows.

We say that a mapping $f: \Omega \rightarrow \mathbf{R}^{n}, \Omega \subset \mathbf{R}^{n}$ is 1-quasiregular if

- $f \in W_{\text {loc }}^{1, n}\left(\Omega, \mathbf{R}^{n}\right)$,

- $D f^{T}(x) D f(x)=|J f(x)|^{\frac{2}{n}}$. I a.e., and

- $J f \geq 0$ a.e or $J f \leq 0$ a.e.

If in addition, $f$ is a homeomorphism, we say that $f$ is 1-quasiconformal.

The purpose of the paper is to provide a new proof of the following version of the Liouville's theorem. The result under such assumptions has been proved originally by Gehring [8].

Theorem 2.1. Let $f: \Omega \rightarrow \mathbf{R}^{n}$ be a 1-quasiconformal mapping in a domain $\Omega \subset \mathbf{R}^{n}, n \geq 3$. Then $f$ is a Möbius transformation in $\mathbf{R}^{n}$ restricted to $\Omega$.

Remark 2.2. Note that the above result implies Reshetnyak's result for 1quasiregular mappings [21], because 1-quasiregular mappings are local homeomorphisms outside a closed branch set of measure zero [3].

To begin, we will need the following basic properties of 1-quasiconformal mappings:

(1) 1-quasiconformal mappings are differentiable a.e.

(2) The Jacobian of a 1-quasiconformal mapping is nonzero a.e. 
(3) The inverse of a 1-quasiconformal mappings is 1-quasiconformal, and the composition of 1-quasiconformal mappings is 1-quasiconformal.

(4) 1-quasiconformal mappings have the Lusin property, i.e. they map sets of Lebesgue measure zero onto sets of Lebesgue measure zero.

For a proof of properties (1) and (2), see [14, Corollary 6.1.1] and [22, p. 216]. Property (3) is immediate from the geometric definition of quasiconformal mappings [8]. Property (4) follows from the fact that any $W^{1, n}$ homeomorphism has the Lusin property [19].

Let $V=f(\Omega)$ and let

$$
g:=f^{-1}: V \rightarrow \Omega,
$$

be the inverse mapping. From the above properties, $g$ is 1-quasiconformal and differentiable a.e. Hence $I=D(g(f(x)))=(D g)(f(x)) D f(x)$ a.e. Thus

$$
(D g)(f(x))=[D f(x)]^{-1} \text { a.e. }
$$

Note that here we use the fact that both $f$ and $g$ have the Lusin property and $J f \neq 0$ a.e.

Fix $e_{i}=(0, \ldots, 1, \ldots, 0)$ and for a compactly contained domain $A \Subset \Omega$ define

$$
f_{t}(x):=g\left(f(x)+t e_{i}\right)
$$

for $x \in A$ and $|t|<\operatorname{dist}(f(A), \partial V)$. It is again a well defined 1-quasiconformal mapping.

Note that for a.e. $x \in \Omega$ we have

$$
\lim _{t \rightarrow 0} \frac{f_{t}(x)-f_{0}(x)}{t}=\lim _{t \rightarrow 0} \frac{g\left(f(x)+t e_{i}\right)-x}{t}=D g(f(x)) e_{i}=[D f(x)]^{-1} e_{i} .
$$

The proof of Theorem 2.1 is based on the following result which is of independent interest.

Theorem 2.3. Let $f: \Omega \rightarrow \mathbf{R}^{n}$, where $\Omega$ is a domain in $\mathbf{R}^{n}$, be 1-quasiconformal and $g=f^{-1}$. For a compact domain $A \Subset \Omega$ define $f_{t}(x):=g\left(f(x)+t e_{i}\right)$ for $x \in A$ and $|t|<\operatorname{dist}(f(A), \partial V)$. Let

$$
X(x):=\lim _{t \rightarrow 0} \frac{f_{t}(x)-f_{0}(x)}{t}=[D f(x)]^{-1} e_{i} .
$$

Then

$$
X \in W_{\text {loc }}^{1,1}\left(\Omega, \mathbf{R}^{n}\right)
$$

and

$$
D X+D X^{T}=\left(\frac{2}{n} \operatorname{div} X\right) \cdot \mathrm{I}
$$

Note that in dimension 2, these are exactly the Cauchy-Riemann equations.

According to Ahlfors' deformation theorem (Theorem 3.3) every distributional vector field that satisfies (2.1) is a polynomial of degree 2. This will allow us to complete the proof of Theorem 2.1 by adapting the argument of Sarvas [23] that he originally used in the $C^{2}$ case. 


\section{Auxiliary results}

In this section we will recall known results that we will need later. For the sake of completeness we provide short proofs.

If $A$ is a square matrix and $A^{\#}$ is the matrix of cofactors, then $A^{T} A^{\#}=(\operatorname{det} A) \cdot \mathrm{I}$. Hence $(D f)^{T}(D f)^{\#}=(J f) \cdot$ I. Thus the Cauchy-Riemann system $(D f)^{T} D f=$ $|J f|^{2 / n} \cdot$ I implies that

$$
(D f)^{\#}= \pm n^{\frac{2-n}{2}}|D f|^{n-2} D f,
$$

where the \pm sign depends on the sign of the Jacobian and $|A|$ stands for the HilbertSchmidt norm of the matrix.

It is well known (see e.g. [14, Lemma 4.8.1]) that for any $u \in W_{\text {loc }}^{1, p}\left(\Omega, \mathbf{R}^{n}\right)$, $\Omega \subset \mathbf{R}^{n}, p \geq n-1$, the matrix of cofactors $(D u)^{\#}$ is divergence free

$$
\operatorname{div}(D u)^{\#}=0 \text {. }
$$

Hence (3.1) yields that any 1-quasiregular mapping is $n$-harmonic

$$
\operatorname{div}\left(|D f|^{n-2} D f\right)=0 .
$$

This is well known. Following the Nireberg method of difference quotients Bojarski and Iwaniec [2] proved the following result. For the sake of completeness we provide a proof.

Theorem 3.1. If $u \in W_{\text {loc }}^{1, p}\left(\Omega, \mathbf{R}^{m}\right), \Omega \subset \mathbf{R}^{n}$ is $p$-harmonic, $p \geq 2$, i.e.

$$
\operatorname{div}\left(|D u|^{p-2} D u\right)=0,
$$

then

$$
|D u|^{(p-2) / 2} D u \in W_{\text {loc }}^{1,2}\left(\Omega, \mathbf{R}^{n \times m}\right) .
$$

Proof. Let $F(x)=|D u(x)|^{(p-2) / 2} D u(x)$. Clearly $F \in L_{\text {loc }}^{2}\left(\Omega, \mathbf{R}^{m \times n}\right)$. According to a difference quotient characterization of $W_{\text {loc }}^{1,2}$ it suffices to prove that for any $\varphi \in C_{0}^{\infty}(\Omega)$

$$
\left(\int_{\Omega} \varphi^{2}(x)|F(x+h)-F(x)|^{2} d x\right)^{1 / 2} \leq C|h| \text { for small } h \in \mathbf{R}^{n} .
$$

Let $G(x)=|D u(x)|^{p-2} D u(x)$. Taking

$$
\psi(x)=\varphi^{2}(x)(u(x+h)-u(x))
$$

as a test function we have

$$
\int_{\Omega}\langle G(x+h)-G(x), D \psi(x)\rangle d x=0
$$

and hence

$$
\begin{aligned}
& \int_{\Omega} \varphi^{2}(x)\langle G(x+h)-G(x), D u(x+h)-D u(x)\rangle d x \\
& =-2 \int_{\Omega} \varphi(x)(u(x+h)-u(x))\langle G(x+h)-G(x), D \varphi(x)\rangle d x .
\end{aligned}
$$

The elementary inequalities for vectors $\xi, \zeta \in \mathbf{R}^{k}$ (valid for $p \geq 2$ )

$$
\begin{aligned}
\left\langle|\xi|^{p-2} \xi-|\zeta|^{p-2} \zeta, \xi-\zeta\right\rangle & \geq\left. C_{1}(p)|| \xi\right|^{(p-2) / 2} \xi-\left.|\zeta|^{(p-2) / 2} \zeta\right|^{2} \\
\left.|| \xi\right|^{p-2} \xi-|\zeta|^{p-2} \zeta \mid & \leq\left. C_{2}(p)\left(|\xi|^{p}+|\zeta|^{p}\right)^{(p-2) /(2 p)}|| \xi\right|^{(p-2) / 2} \xi-|\zeta|^{(p-2) / 2} \zeta \mid
\end{aligned}
$$


applying to matrices regarded as vectors give

$$
\begin{aligned}
& \int_{\Omega} \varphi^{2}(x)|F(x+h)-F(x)|^{2} d x \\
& \leq C \int_{\Omega}|\varphi(x)||u(x+h)-u(x)||D \varphi(x)| \\
& \quad \cdot\left(|D u(x+h)|^{p}+|D u(x)|^{p}\right)^{(p-2) /(2 p)}|F(x+h)-F(x)| d x . \\
& \leq C\left(\int_{\Omega}|\varphi(x)|^{2}|F(x+h)-F(x)|^{2} d x\right)^{1 / 2} \\
& \quad \cdot\left(\int_{\Omega}|u(x+h)-u(x)|^{2}|D \varphi(x)|^{2}\left(|D u(x+h)|^{p}+|D u(x)|^{p}\right)^{(p-2) / p} d x\right)^{1 / 2} .
\end{aligned}
$$

Thus

$$
\begin{aligned}
& \int_{\Omega} \varphi^{2}(x)|F(x+h)-F(x)|^{2} d x \\
& \leq C \int_{\Omega}|u(x+h)-u(x)|^{2}|D \varphi(x)|^{2}\left(|D u(x+h)|^{p}+|D u(x)|^{p}\right)^{(p-2) / p} d x \\
& \leq C\left(\int_{\Omega}|u(x+h)-u(x)|^{p}|D \varphi(x)|^{p} d x\right)^{2 / p}\left(\int_{\operatorname{supp} \varphi}|D u(x+h)|^{p}+|D u(x)|^{p} d x\right)^{(p-2) / p}
\end{aligned}
$$

and it suffices to observe that the first integral on the right hand side is bounded by $C|h|^{2}$, while the second integral is bounded by a constant independent of (small) $h$. $s \leq p$,

Corollary 3.2. If $u \in W_{\mathrm{loc}}^{1, p}\left(\Omega, \mathbf{R}^{m}\right)$ is $p$-harmonic, $p \geq 2$, then for any $p / 2 \leq$

$$
|D u|^{s-1} D u \in W_{\text {loc }}^{1, p / s} .
$$

Proof. For $s=p / 2$ this is the previous result, so we can assume that $p / 2<s \leq p$. The matrix function

$$
\Phi_{\alpha}(A)=|A|^{\alpha} A, \quad \alpha>0
$$

is of class $C^{1}$ and

$$
|D u|^{s-1} D u=\Phi_{\frac{2 s-p}{p}}\left(|D u|^{(p-2) / 2} D u\right) .
$$

Since $|D u|^{(p-2) / 2} D u \in W_{\text {loc }}^{1,2}$, the result follows from the chain rule.

Let now $f$ be 1-quasiconformal and $g=f^{-1}$ be the inverse mapping. Then $g$ is also 1-quasiconformal and hence $n$-harmonic. Thus the above corollary implies that

$$
\pm n^{\frac{n-2}{2}}(D g)^{\#}=|D g|^{n-2} D g \in W_{\mathrm{loc}}^{1, \frac{n}{n-1}}
$$

and

$$
J g= \pm|J g|= \pm n^{-\frac{n}{2}}|D g|^{n} \in W_{\text {loc }}^{1,1} .
$$

The next result is a variant of the Ahlfors deformation theorem [1], where the original version assumes the vector field is $C^{1}$. However, by approximating distributions by Schwarz functions one easily sees that these two versions are actually equivalent. 
Theorem 3.3. If $X$ is a distributional vector field in a domain $\Omega \subset \mathbf{R}^{n}, n \geq 3$, which satisfies

$$
D X+D X^{T}=\left(\frac{2}{n} \operatorname{div} X\right) \cdot \mathrm{I},
$$

then $X$ is a polynomial of degree 2 and is of the form

$$
X(x)=a+B x+2\langle c, x\rangle x-|x|^{2} c
$$

where $a, c \in \mathbf{R}^{n}$ and $B=\left[b_{i j}\right]: \mathbf{R}^{n} \rightarrow \mathbf{R}^{n}$ is a linear mapping satisfying $b_{i j}=-b_{j i}$ for $i \neq j$ and $b_{i i}=b_{j j}$ for all $i, j$.

Proof. In order to prove that $X$ is a polynomial of degree 2 it suffices to show that all distributional partial derivatives of order 3 are equal zero.

Let $X=\left(X_{1}, \ldots, X_{n}\right), X_{i, j}=\frac{\partial}{\partial x_{j}} X_{i}, X_{i, j k}=\frac{\partial}{\partial x_{k}} X_{i, j}$ in the distributional sense, and so on. From (3.4) one immediately gets that $X_{i, j}=-X_{j, i}$ for $i \neq j$, and $X_{i, i}=X_{j, j}$ for all $i, j$.

Since $n \geq 3$ we take $i, j, k$ distinct and then,

$$
X_{i, j k}=X_{i, k j}=-X_{k, i j}=-X_{k, j i}=X_{j, k i}=X_{j, i k}=-X_{i, j k} .
$$

Hence $X_{i, j k}=0$ for $i, j, k$ distinct.

We will show that $X_{i, j k \ell}=0$ for all $i, j, k, \ell$. If we have at least 3 distinct indices among $\{i, j, k, \ell\}$, we can always permute them to have the first three indices distinct and $X_{i, j k \ell}=0$ is obvious. If there are only two distinct indices, say, $\{i, j, k, \ell\}=$ $\{i, j\}, i \neq j$, then we have two cases $X_{i, i j j}$ and $X_{i, j j j}$ (plus permutation of indices). We have

$$
X_{i, i j j}=X_{i, j i j}=-X_{j, i i j}=-X_{j, j i i} .
$$

Since $n \geq 3$, there is $k$ different from $i, j$ and hence

$$
X_{i, i j j}=-X_{j, j i i}=-X_{k, k i i}=X_{i, i k k}=X_{j, j k k}=-X_{k, k j j}=-X_{i, i j j}=0,
$$

where we repeatedly use (3.5). In the case $X_{i, j j j}$, we again find $k$ different from $i, j$

$$
X_{i, j j j}=-X_{j, i j j}=-X_{j, j i j}=-X_{k, k i j}=-X_{k, i j k}=0 .
$$

The last case is when all indices are equal, but in that case

$$
X_{i, i i i}=X_{j, j i i}=0
$$

by the case proved above.

Thus $X$ is a polynomial of degree 2 and hence

$$
X_{i}=a_{i}+\sum_{j} b_{i j} x_{j}+\sum_{j, k} c_{i j k} x_{j} x_{k} .
$$

We may assume $c_{i j k}=c_{i k j}$. Thus

$$
X_{i, j}=b_{i j}+2 \sum_{k} c_{i j k} x_{k}, \quad X_{i, j k}=c_{i j k} .
$$

Since $X_{i, j}=-X_{j, i}$ for $i \neq j$ and $X_{i, i}=X_{j, j}$ for all $i, j, b_{i j}=-b_{j i}$ for $i \neq j$ and $b_{i i}=b_{j j}$ for all $i, j$. 
If $i, j, k$ are distinct, then $c_{i j k}=X_{i, j k}=0$, so

$$
\begin{aligned}
X_{i} & =a_{i}+\sum_{j} b_{i j} x_{j}+\sum_{k} c_{i i k} x_{i} x_{k}+\sum_{k \neq i} c_{i k i} x_{k} x_{i}+\sum_{k \neq i} c_{i k k} x_{k}^{2} \\
& =a_{i}+\sum_{j} b_{i j} x_{j}+2 \sum_{k} c_{i i k} x_{i} x_{k}-c_{i i i} x_{i}^{2}+\sum_{k \neq i} c_{i k k} x_{k}^{2} .
\end{aligned}
$$

Since $X_{i, i}=X_{j, j}$ for all $i, j$,

$$
c_{i i k}=c_{j j k}:=c_{k} \text { for all } i, j, k,
$$

and since $X_{i, k}=-X_{k, i}$ for $i \neq k$,

$$
c_{i k k}=-c_{k i k}=-c_{k k i}=-c_{i} \text { for } i \neq k .
$$

Thus

$$
\begin{aligned}
X_{i} & =a_{i}+\sum_{j} b_{i j} x_{j}+2\left(\sum_{k} c_{k} x_{k}\right) x_{i}-c_{i} \sum_{k} x_{k}^{2} \\
& =a_{i}+\sum_{j} b_{i j} x_{j}+2\langle c, x\rangle x_{i}-|x|^{2} c_{i} .
\end{aligned}
$$

The proof is complete.

\section{Proof of Theorem 2.3}

Recall that $f: \Omega \rightarrow \mathbf{R}^{n}$, where $\Omega$ is a domain in $\mathbf{R}^{n}$, is 1-quasiconformal. Let $V=f(\Omega)$ and $g:=f^{-1}: V \rightarrow \Omega$. For a compact domain $A \Subset \Omega$ define $f_{t}(x):=$ $g\left(f(x)+t e_{i}\right)$ for $x \in A$ and $|t|<\operatorname{dist}(f(A), \partial V)$. Let

$$
X_{t}(x):=\frac{f_{t}(x)-x}{t} \in W_{\mathrm{loc}}^{1, n}(\Omega) .
$$

We know $X_{t} \rightarrow X=[D f]^{-1} e_{i}$ a.e. Furthermore, we claim that

Lemma 4.1. For every compact set $A \subset \Omega$

$$
X_{t}(x)=\frac{f_{t}(x)-x}{t} \rightarrow X(x) \text { in } L^{1}(A) \text { as } t \rightarrow 0 .
$$

Proof. Since we have a.e. convergence, by a generalized version of Dominated Convergence theorem ([6], Theorem 21, p. 23), the above result follows easily from the following lemma.

Lemma 4.2. The family of functions $X_{t}(x)$ is equi-integrable in any compact subset of $\Omega$.

Proof. We first note that by (3.3) and the Sobolev embedding theorem $J g \in$ $L_{\text {loc }}^{\frac{n}{n-1}}(V)$. Let $A$ be a compact set of $\Omega$ and $E$ be any measurable subset of $A$. Since $g$ is 1-quasiconformal and thus has Lusin property, we can apply change of variable formula [10] to obtain

$$
\begin{aligned}
& \int_{E}\left|\frac{f_{t}(x)-x}{t}\right| d x=\int_{f(E)}\left|\frac{g\left(y+t e_{i}\right)-g(y)}{t}\right||J g(y)| d y \\
& \leq\left\|\frac{g\left(y+t e_{i}\right)-g(y)}{t}\right\|_{L^{n}(f(E))}\|J g\|_{L^{\frac{n}{n-1}}(f(E))} \leq M\|J g\|_{L^{\frac{n}{n-1}}(f(E))},
\end{aligned}
$$


because $g \in W_{\text {loc }}^{1, n}$ and hence the difference quotients of $g$ are bounded in $L^{n}$ on compact subsets of $V$. Let $\varepsilon>0$ be given, since $J g \in L^{\frac{n}{n-1}}(f(A))$, by absolute continuity of the integral, there is $c>0$ such that $\|J g\|_{L^{n /(n-1)}(f(E))}<\varepsilon M^{-1}$ whenever $|f(E)|<c$. Since $|f(E)|=\int_{E}|J f| d x$, there is $\delta>0$ such that $|f(E)|<c$ whenever $|E|<\delta$. Thus, for $|E|<\delta$, the left hand side of (4.1) is less than $\varepsilon$. The proof is complete.

Now we will prove that the derivatives of $X_{t}$,

$$
D X_{t}=\frac{D f_{t}-\mathrm{I}}{t} \in L_{\mathrm{loc}}^{n}(\Omega)
$$

converge in the distributional sense to a function in $L_{\text {loc }}^{1}$.

Lemma 4.3. There exists $u \in L_{\text {loc }}^{1}\left(\Omega, \mathbf{R}^{n \times n}\right)$ such that

$$
\int_{\Omega} D X_{t}(x) \varphi(x) d x \rightarrow \int_{\Omega} u(x) \varphi(x) d x
$$

as $t \rightarrow 0$ for all $\varphi \in C_{0}^{\infty}(\Omega)$.

Proof. Without loss of generality we may assume that $J g \geq 0$ a.e. By the change of variables,

$$
\begin{aligned}
\int_{\Omega} \frac{D f_{t}(x)-\mathrm{I}}{t} \varphi(x) d x & =\int_{\Omega} \frac{D g\left(f(x)+t e_{i}\right) D f(x)-\mathrm{I}}{t} \varphi(x) d x \\
& =\int_{V} \frac{D g\left(y+t e_{i}\right) D f(g(y))-\mathrm{I}}{t} J g(y) \varphi(g(y)) d y \\
& =\int_{V} \frac{D g\left(y+t e_{i}\right)[D g(y)]^{-1}-\mathrm{I}}{t} J g(y) \varphi(g(y)) d y
\end{aligned}
$$

for $D f(g(y))=[D g(y)]^{-1}$ a.e. From the formula for the inverse matrix we have that $[D g(y)]^{-1} J g(y)=\left(D g^{\#}(y)\right)^{T}$ if $J g(y) \neq 0$. Hence (4.2) is equal to,

$$
\begin{aligned}
& \int_{V} \frac{D g\left(y+t e_{i}\right)\left(D g^{\#}(y)\right)^{T}-J g(y) \cdot \mathrm{I}}{t} \varphi(g(y)) d y \\
& =\int_{V} D g\left(y+t e_{i}\right) \frac{\left[\left(D g^{\#}(y)\right)-D g^{\#}\left(y+t e_{i}\right)\right]^{T}}{t} \varphi(g(y)) d y \\
& \quad+\int_{V} \frac{J g\left(y+t e_{i}\right)-J g(y)}{t} \cdot \mathrm{I} \varphi(g(y)) d y .
\end{aligned}
$$

The last equality follows from $D g(y)\left(D g^{\#}\right)^{T}(y)=J g(y) \cdot \mathrm{I}$.

Since by (3.2), $D g^{\#} \in W_{\text {loc }}^{1, \frac{n}{n-1}}(V)$, it is an elementary fact, [9, p. 265], that

$$
\frac{D g^{\#}(y)-D g^{\#}\left(y+t e_{i}\right)}{t} \rightarrow-\frac{\partial}{\partial y_{i}} D g^{\#}(y) \quad \text { in } L_{\mathrm{loc}}^{\frac{n}{n-1}}(V)
$$

On the other hand, $D g \in L_{\text {loc }}^{n}(V), D g\left(y+t e_{i}\right)$ is a translation of $D g(y)$ and $\varphi(g(y))$ is bounded with compact support, so $D g\left(y+t e_{i}\right) \varphi(g(y)) \rightarrow D g(y) \varphi(g(y))$ in $L^{n}(V)$ as $t \rightarrow 0$. We thus obtain convergence for the first integral on the right hand side 
of $(4.3)$

$$
\begin{aligned}
& \int_{V} D g\left(y+t e_{i}\right) \frac{\left[\left(D g^{\#}(y)\right)-D g^{\#}\left(y+t e_{i}\right)\right]^{T}}{t} \varphi(g(y)) d y \\
& \rightarrow-\int_{V} D g(y)\left[\frac{\partial}{\partial y_{i}} D g^{\#}(y)\right]^{T} \varphi(g(y)) d y .
\end{aligned}
$$

Since by (3.3), $J g(y) \in W_{\text {loc }}^{1,1}(V)$,

$$
\frac{J g\left(y+t e_{i}\right)-J g(y)}{t} \rightarrow \frac{\partial}{\partial y_{i}} J g(y) \text { in } L_{\mathrm{loc}}^{1}(V) .
$$

Hence we obtain convergence for the second integral on the right hand side of (4.3)

$$
\int_{V} \frac{J g\left(y+t e_{i}\right)-J g(y)}{t} \cdot \mathrm{I} \varphi(g(y)) d y \rightarrow \int_{V} \frac{\partial}{\partial y_{i}} J g(y) \cdot \mathrm{I} \varphi(g(y)) d y .
$$

Thus

$$
\begin{aligned}
\int_{\Omega} \frac{D f_{t}(x)-\mathrm{I}}{t} \varphi(x) d x \rightarrow- & \int_{V} D g(y)\left[\frac{\partial}{\partial y_{i}} D g^{\#}(y)\right]^{T} \varphi(g(y)) d y \\
& +\int_{V} \frac{\partial}{\partial y_{i}} J g(y) \cdot \mathrm{I} \varphi(g(y)) d y=\int_{\Omega} u(x) \varphi(x) d x
\end{aligned}
$$

where

$$
u(x)=\left[-D g\left(f(x)\left[\left(\frac{\partial}{\partial y_{i}} D g^{\#}\right)(f(x))\right]^{T}+\left(\frac{\partial}{\partial y_{i}} J g\right)(f(x)) \cdot \mathrm{I}\right] J f(x) \in L_{\mathrm{loc}}^{1}(\Omega),\right.
$$

since

$$
-D g(y)\left[\frac{\partial}{\partial y_{i}} D g^{\#}(y)\right]^{T}+\frac{\partial}{\partial y_{i}} J g(y) \cdot \mathrm{I} \in L_{\mathrm{loc}}^{1}(V) .
$$

The proof is complete.

Corollary 4.4. $D X=u \in L_{\text {loc }}^{1}$ and hence $X \in W_{\text {loc }}^{1,1}(\Omega)$.

Proof. By Lemma 4.1 and 4.3,

$$
\begin{aligned}
\int_{\Omega} X(x) \frac{\partial \varphi}{\partial x_{j}}(x) d x & =\lim _{t \rightarrow 0} \int_{\Omega} X_{t}(x) \frac{\partial \varphi}{\partial x_{j}}(x) d x=-\lim _{t \rightarrow 0} \int_{\Omega} \frac{\partial X_{t}}{\partial x_{j}}(x) \varphi(x) d x \\
& =-\lim _{t \rightarrow 0} \int_{\Omega} D X_{t}(x) e_{j} \varphi(x) d x=-\int_{\Omega} u(x) e_{j} \varphi(x) d x
\end{aligned}
$$

Thus $D X=u \in L_{\text {loc }}^{1}$. The proof is complete.

Since

$$
D f_{t}^{T} D f_{t}=J f_{t}^{2 / n} \cdot \mathrm{I} \text { a.e., and } J f_{t}>0 \text { a.e., }
$$

we have

$$
\frac{J f_{t}^{1 / n}-1}{t} \cdot \mathrm{I}=\frac{\frac{D f_{t}^{T} D f_{t}}{J f_{t}^{1 / n}}-\mathrm{I}}{t}=\frac{\left(D f_{t}-J f_{t}^{1 / n} \cdot \mathrm{I}\right)^{T} D f_{t}}{t J f_{t}^{1 / n}}+\frac{D f_{t}-\mathrm{I}}{t} .
$$

Observe that

$$
\frac{\left(D f_{t}-J f_{t}^{1 / n} \cdot \mathrm{I}\right)^{T} D f_{t}}{t J f_{t}^{1 / n}}=\frac{J f_{t}^{1 / n}-1}{t} \cdot \mathrm{I}-\frac{D f_{t}-\mathrm{I}}{t} \in L_{\mathrm{loc}}^{n} .
$$


Lemma 4.5. There exists $v(x) \in L_{\mathrm{loc}}^{1}(\Omega)$ such that for all $\varphi \in C_{0}^{\infty}(\Omega)$

$$
\int_{\Omega} \frac{\left(D f_{t}-J f_{t}^{1 / n} \cdot \mathrm{I}\right)^{T} D f_{t}}{t J f_{t}^{1 / n}} \varphi(x) d x \rightarrow \int_{\Omega} u^{T}(x) \varphi(x) d x-\int_{\Omega} v(x) \cdot \mathrm{I} \varphi(x) d x
$$

as $t \rightarrow 0$, where $u$ is the same as in Lemma 4.3.

Proof. Recall that

$$
D f_{t}(x)=D g\left(f(x)+t e_{i}\right) D f(x), \quad J f_{t}(x)=J g\left(f(x)+t e_{i}\right) J f(x),
$$

and $D f(g(y))=[D g(y)]^{-1}$. Hence the change of variables formula yields

$$
\begin{aligned}
\int_{\Omega} & \frac{\left(D f_{t}-J f_{t}^{1 / n} \cdot \mathrm{I}\right)^{T} D f_{t}}{t J f_{t}^{1 / n}} \varphi(x) d x=\int_{V} \varphi(g(y)) J g(y) \\
& \cdot \frac{\left(D g\left(y+t e_{i}\right)[D g(y)]^{-1} J g(y)^{\frac{1}{n}}-J g\left(y+t e_{i}\right)^{\frac{1}{n}} \cdot \mathrm{I}\right)^{T} D g\left(y+t e_{i}\right)[D g(y)]^{-1}}{t J g\left(y+t e_{i}\right)^{\frac{1}{n}}} d y .
\end{aligned}
$$

Since

$$
[D g]^{-1} J g=\left(D g^{\#}\right)^{T}, \quad D g^{T} D g=J g^{\frac{2}{n}} \cdot \mathrm{I}, \quad[D g]^{-1}=D g^{T} / J g^{\frac{2}{n}},
$$

one easily checks the above is equal to,

$$
\begin{aligned}
\int_{V} \varphi(g(y))\left[\frac{\left[\left(D g^{\#}(y)-D g^{\#}\left(y+t e_{i}\right)\right] J g\left(y+t e_{i}\right)^{\frac{1}{n}} D g^{T}(y)\right.}{t J g(y)^{\frac{1}{n}}}\right. & \\
& \left.+\frac{\left[J g\left(y+t e_{i}\right)^{1-\frac{1}{n}}-J g(y)^{1-\frac{1}{n}}\right] D g\left(y+t e_{i}\right) D g^{T}(y)}{t J g(y)^{\frac{1}{n}}}\right] d y .
\end{aligned}
$$

We know from the proof of Lemma 4.3 that

$$
\frac{D g^{\#}(y)-D g^{\#}\left(y+t e_{i}\right)}{t} \rightarrow-\frac{\partial}{\partial y_{i}} D g^{\#}(y) \text { in } L_{\text {loc }}^{\frac{n}{n-1}} .
$$

We will show now that

$$
\frac{J g\left(y+t e_{i}\right)^{\frac{1}{n}} D g^{T}(y)}{J g(y)^{\frac{1}{n}}} \rightarrow D g^{T}(y) \quad \text { in } L_{\mathrm{loc}}^{n}(V) .
$$

Indeed, $n^{\frac{n}{2}} J g(y)=|D g|^{n}$, so $\left|D g(y) J g(y)^{-\frac{1}{n}}\right|=n^{\frac{1}{2}}$. Hence for any compact set $K \subset V$,

$$
\begin{aligned}
& \int_{K}\left|\frac{J g\left(y+t e_{i}\right)^{\frac{1}{n}} D g^{T}(y)}{J g(y)^{\frac{1}{n}}}-D g^{T}(y)\right|^{n} d y \\
& \leq \int_{K}\left|J g\left(y+t e_{i}\right)^{\frac{1}{n}}-J g(y)^{\frac{1}{n}}\right|^{n}\left|D g^{T}(y) J g(y)^{-\frac{1}{n}}\right|^{n} d y \\
& =n^{\frac{n}{2}} \int_{K}\left|J g\left(y+t e_{i}\right)^{\frac{1}{n}}-J g(y)^{\frac{1}{n}}\right|^{n} d y \rightarrow 0 .
\end{aligned}
$$

This implies convergence of the first half of (4.8),

$$
\begin{aligned}
& \int_{V} \varphi(g(y)) \frac{\left[\left(D g^{\#}(y)-D g^{\#}\left(y+t e_{i}\right)\right] J g\left(y+t e_{i}\right)^{\frac{1}{n}} D g^{T}(y)\right.}{t J g(y)^{\frac{1}{n}}} d y \\
& \rightarrow-\int_{V} \varphi(g(y))\left[\frac{\partial}{\partial y_{i}} D g^{\#}(y)\right] D g^{T}(y) d y
\end{aligned}
$$


By (3.2) and (3.3) $J g(y)^{1-\frac{1}{n}}=c\left|D g^{\#}(y)\right| \in W_{\mathrm{loc}}^{1, \frac{n}{n-1}}(V)$, thus,

$$
\frac{J g\left(y+t e_{i}\right)^{1-\frac{1}{n}}-J g(y)^{1-\frac{1}{n}}}{t} \rightarrow \frac{\partial}{\partial y_{i}}\left[J g(y)^{1-\frac{1}{n}}\right] \text { in } L_{\text {loc }}^{\frac{n}{n-1}}(V) .
$$

And by the same argument as in (4.9),

$$
D g\left(y+t e_{i}\right) D g^{T}(y) J g(y)^{-\frac{1}{n}} \rightarrow J g(y)^{\frac{1}{n}} \cdot \mathrm{I} \quad \text { in } L_{\mathrm{loc}}^{n}(V) .
$$

Hence we have convergence for the second half of (4.8),

$$
\begin{aligned}
& \int_{V} \varphi(g(y)) \frac{J g\left(y+t e_{i}\right)^{1-\frac{1}{n}}-J g(y)^{1-\frac{1}{n}}}{t} D g\left(y+t e_{i}\right) D g^{T}(y) J g(y)^{-\frac{1}{n}} d y \\
& \rightarrow \int_{V} \varphi(g(y)) \frac{\partial}{\partial y_{i}}\left[J g(y)^{1-\frac{1}{n}}\right] J g(y)^{\frac{1}{n}} \cdot \mathrm{I} d y=\frac{n-1}{n} \int_{V} \varphi(g(y)) \frac{\partial}{\partial y_{i}} J g(y) \cdot \mathrm{I} d y .
\end{aligned}
$$

The last equality follows from $J g(y)=\left[J g(y)^{1-\frac{1}{n}}\right]^{\frac{n}{n-1}}$ and the chain rule for Sobolev functions. Now (4.8), (4.10) and (4.11) yield

$$
\begin{aligned}
& \int_{\Omega} \frac{\left(D f_{t}-J f_{t}(x)^{1 / n} \cdot \mathrm{I}\right)^{T} D f_{t}}{t J f_{t}(x)^{1 / n}} \varphi(x) d x \\
& \rightarrow-\int_{V} \varphi(g(y))\left[\frac{\partial}{\partial y_{i}} D g^{\#}(y)\right] D g^{T}(y) d y+\frac{n-1}{n} \int_{V} \varphi(g(y)) \frac{\partial}{\partial y_{i}} J g(y) \cdot \mathrm{I} d y \\
& \quad=\int_{\Omega} u^{T}(x) \varphi(x) d x-\int_{\Omega} v(x) \cdot \mathrm{I} \varphi(x) d x
\end{aligned}
$$

where $u(x) \in L_{\text {loc }}^{1}(\Omega)$ is the same matrix valued function as in Lemma 4.3 and $v(x)=$ $\frac{1}{n}\left(\frac{\partial}{\partial y_{i}} J g\right)(f(x)) J f(x) \in L_{\text {loc }}^{1}(\Omega)$ is a scalar function. The proof is complete.

Recall that

$$
\frac{J f_{t}^{1 / n}-1}{t} \cdot \mathrm{I}=\frac{\left(D f_{t}-J f_{t}^{1 / n} \cdot \mathrm{I}\right)^{T} D f_{t}}{t J f_{t}^{1 / n}}+\frac{D f_{t}-\mathrm{I}}{t} .
$$

As Lemma 4.3 and 4.5 show

$$
\frac{\left(D f_{t}-J f_{t}^{1 / n} \cdot \mathrm{I}\right)^{T} D f_{t}}{t J f_{t}^{1 / n}}+\frac{D f_{t}-\mathrm{I}}{t} \rightarrow u^{T}-v \cdot \mathrm{I}+u .
$$

in distribution. This forces the left hand side to converge to some $L_{\text {loc }}^{1}\left(\Omega, \mathbf{R}^{n \times n}\right)$ function in distribution as well. However, since the off-diagonal terms of $\left(J f_{t}^{1 / n}-1\right) / t$. I are zero, the limiting function must then be of the form $w \cdot$ I for some $w \in L_{\text {loc }}^{1}(\Omega)$. Recall by Corollary 4.4 that $D X=u$, hence,

$$
D X^{T}-v \cdot \mathrm{I}+D X=w \cdot \mathrm{I} .
$$

Hence $2 X_{i, i}=v+w, i=1,2, \ldots, n$, so $\operatorname{div} X=n / 2(v+w)$. We then conclude

$$
D X+D X^{T}=\left(\frac{2}{n} \operatorname{div} X\right) \cdot \mathrm{I} .
$$

The proof of Theorem 2.3 is complete. 


\section{Proof of Theorem 2.1}

Once we obtain Theorem 2.3, the proof of the Liouville Theorem follows from any well-known proofs under $C^{3}$ or $C^{4}$ assumption. Indeed, Theorem 3.3 tells us that $[D f]^{-1}(x) e_{i}$ is $C^{\infty}$ smooth for every $i=1, \ldots, n$, hence by conformality,

$$
\frac{1}{J f^{2 / n}}=\left\langle[D f]^{-1} e_{i},[D f]^{-1} e_{i}\right\rangle
$$

is also smooth and is a polynomial of degree 4 . Let $\tilde{\Omega}$ be the open subset of $\Omega$ with the roots of $1 /\left(J f^{2 / n}\right)$ removed. It then follows $J f$ is smooth in the open set $\tilde{\Omega}$. Now by conformality again, $D f^{T}=[D f]^{-1} J f^{2 / n}$ is $C^{\infty}$ smooth in $\tilde{\Omega}$. We can then apply, say Nevanlinna's argument [20] to obtain that $f$ is a Möbius transformation in $\tilde{\Omega}$. By the fact that $f$ is a homeomorphism in $\Omega$ we can actually conclude that $f$ is a Möbius transformation in $\Omega$.

However, here we also provide another interesting proof due to Sarvas [23]: Given $f$ 1-quasiconformal, we can assume $0 \in \Omega, f(0)=0$, and $D f(0)=\mathrm{I}$. Indeed, we can compose $f$ with translations and dilation and note that the composition is again a 1-quasiconformal mapping. Therefore, $f(x)=x+|x| \epsilon(x)$ with $\epsilon(x) \rightarrow 0$ as $x \rightarrow 0$. Let $h(x)=\frac{x}{|x|^{2}}$ be an inversion with respect to the unit sphere. Let $\tilde{\Omega}=\Omega \backslash\{0\}$. $\tilde{\Omega}$ is open and $f(x) \neq 0$ on $\tilde{\Omega}$. Then $F: \tilde{\Omega} \rightarrow \mathbf{R}^{n}, F=h \circ f$ is also 1-quasiconformal. Now $D F(x)=|f(x)|^{-2}\left(\mathrm{I}-2 Q_{f(x)}\right) D f(x), x \neq 0$, where $Q_{x}: \mathbf{R}^{n} \rightarrow$ $\mathbf{R}^{n}$ is given by $Q_{x} y=|x|^{-2}\langle y, x\rangle x$ and $\left(\mathrm{I}-2 Q_{x}\right)^{-1}=\left(\mathrm{I}-2 Q_{x}\right)$. Hence $[D F(x)]^{-1} e_{i}=$ $|f(x)|^{2}[D f(x)]^{-1}\left(\mathrm{I}-2 Q_{f(x)}\right) e_{i}=|f(x)|^{2}[D f(x)]^{-1} e_{i}-2[D f(x)]^{-1}\left\langle f(x), e_{i}\right\rangle f(x)$. Note that $[D f(x)]^{-1} e_{i}$ is a polynomial of degree 2. In particular, it is defined for $x=0$ since $D f(0)=\mathrm{I}$ and $f$ a homeomorphism with $f(0)=0$, hence $[D F(0)]^{-1} e_{i}=0$. Now since by Theorem 3.3, $[D F(x)]^{-1} e_{i}=a+B x+2\langle c, x\rangle x-|x|^{2} c$. The condition $[D F(0)]^{-1} e_{i}=0$ gives $a=0$. Let $e \in \mathbf{R}^{n},|e|=1$. Inserting $x=$ se for small $s>0$, we get

$$
\begin{aligned}
s B e=B(s e) & =-s^{2}[2\langle c, e\rangle e-c]+[D F(s e)]^{-1} e_{i} \\
& =-s^{2}[2\langle c, e\rangle e-c]+|f(s e)|^{2}[D f(s e)]^{-1}\left(\mathrm{I}-2 Q_{f(s e)}\right) e_{i} .
\end{aligned}
$$

Substituting $f(x)=x+|x| \epsilon(x)$ we get

$$
s B e=-s^{2}[2\langle c, e\rangle e-c]+s^{2}|e+\epsilon(s e)|^{2}\left([D f(s e)]^{-1}\left(\mathrm{I}-2 Q_{f(s e)}\right) e_{i} .\right.
$$

Dividing by $s$ and let $s \rightarrow 0$ gives $B e=0$. Since $e$ is an arbitrary unit vector, this yields $B=0$. Therefore $[D F(x)]^{-1} e_{i}=2\langle c, x\rangle x-|x|^{2} c$. This implies $c$ cannot be zero. Otherwise, $D F^{-1}=0$ everywhere, violating $J(x, F) \neq 0$ a.e. for quasiconformal mappings. Putting $x=s c$ for small $s>0$ we obtain,

$$
s^{2}|c|^{2} c=D F^{-1}(s c) e_{i}=s^{2}|c+| c|\epsilon(s c)|^{2}[D f(s c)]^{-1}\left(\mathrm{I}-2 Q_{f(s c)}\right) e_{i} .
$$

Divide by $s^{2}$ and then let $s \rightarrow 0$, then $Q_{f(s c)} e_{i} \rightarrow Q_{c} e_{i}$ since $f(x)=x+|x| \epsilon(x)$, and so the above implies $c=\left(\mathrm{I}-2 Q_{c}\right) e_{i}$, and this implies $e_{i}=\left(\mathrm{I}-2 Q_{c}\right) c=-c$. Finally,

$$
[D F(x)]^{-1} e_{i}=-\left(2\left\langle e_{i}, x\right\rangle x-|x|^{2} e_{i}\right)=|x|^{2}\left(\mathrm{I}-2 Q_{x}\right) e_{i} .
$$

Since $i$ as for $e_{i}$ is arbitrary, we conclude that $[D F(x)]^{-1}=|x|^{2}\left(\mathrm{I}-2 Q_{x}\right)=[D h(x)]^{-1}$ for $x \in \tilde{\Omega}$, or $D F=D h$ for all $x \in \tilde{\Omega}$. Thus $F=h+d$ for some constant vector $d$ and for all $x \in \tilde{\Omega}$. Note that $F=h \circ f$, thus $f(x)=h^{-1}(h(x)+d)=h(h(x)+d)$. In the above argument we do not distinguish a.e. equivalent functions, but this is not a 
problem since $f$ is a homeomorphism so they must equal everywhere. The proof is complete.

Acknowledgements. The author would like to thank Piotr Hajłasz for many useful discussions and suggestions, especially his great efforts in reshaping this paper. The author would also like to thank Paweł Konieczny for bringing the Ahlfors deformation theorem to the author's attention. The author was partially supported by the NSF grant DMS-0900871 of Piotr Hajłasz.

\section{References}

[1] Ahlfors, L. V.: Conditions for quasiconformal deformations in several variables. - Contributions to analysis, Academic Press, New York, 1974.

[2] Bojarski, B., and T. Iwaniec: $p$-harmonic equation and quasiregular mappings. - In: Partial differential equations (Warsaw, 1984), Banach Center Publ. 19, PWN, Warsaw, 1987, 25-38.

[3] Bojarski, B., and T. IwAniec: Analytical foundations of the theory of quasiconformal mappings in $\mathbf{R}^{n}$. - Ann. Acad. Sci. Fenn. Ser. A I Math. 8, 1983, 257-324.

[4] Bojarski, B., and T. Iwaniec: Another approach to Liouville theorem. - Math. Nachr. 107, 1982, 253-262.

[5] Capelli, A.: Sulla limitata possibilitá di trasformazioni conformi nello spazio. - Ann. Mat. Pura Appl. (2) 14, 1886, 227-237.

[6] Dellacherie, C., and P. Meyer: Probabilities and potential. - North-Holland Mathematics Series 29, Amsterdam, 1978.

[7] Do CARmo, M.: Riemannian geometry. - Birkhäuser, Boston, 1992.

[8] Gehring, F. W.: Rings and quasiconformal mappings in space. - Trans. Amer. Math. Soc. 103, 1962, 353-393.

[9] Guisti, E.: Direct methods in the calculus of variations. - World Sci. Publ., 2003.

[10] HajŁasz, P.: Change of variables formula under minimal assumptions. - Colloq. Math. 64, 1993, 93-101.

[11] Hartman, P.: Systems of total differential equations and Liouville's theorem on conformal mappings. - Amer. J. Math. 69, 1947, 327-332.

[12] Hartman, P.: On Isometries and on a theorem of Liouville. - Math. Z. 69, 1958, 202-210.

[13] IWANIEC, T.: p-harmonic tensors and quasiregular mappings. - Ann. of Math. (2) 136, 1992, 589-624.

[14] Iwaniec, T., and G. Martin: Geometric function theory and nonlinear analysis. - Oxford Math. Monogr., The Clarendon Press, Oxford Univ. Press, 2001.

[15] Iwaniec, T., and G. Martin: Quasiregular mappings in even dimensions - Acta. Math. 170, 1993, 29-81.

[16] Iwaniec, T., and G. Martin: The Liouville theorem. - In: Analysis and topology, World Sci. Publ., River Edge, NJ, 1998, 339-361.

[17] Liouville, J.: Extension au cas des trois dimensions da la question du tracégéograhpique. Note VI. - In: Monge, G. (ed.), Applications de l'analysis à la géométrie, Bachelier, Paris, 1850, 609-617.

[18] LiU, Z.: The Liouville theorem under second order differentiability assumption. - Submitted.

[19] Malý, J., and O. Martio: Lusin's condition (N) and mappings of the class $W^{1, n}-$ J. Reine Angew. Math. 458, 1995, 19-36.

[20] Nevanlinna, R.: On differentiable mappings. - In: Analytic functions, Princeton Univ. Press, Princeton, N.J., 1960, 3-9. 
[21] Reshetnyak, YU. G.: Liouville's theorem on conformal mappings for minimal regularity assumptions. - Sib. Math. J. 8, 1967, 631-634.

[22] Reshetnyak, Yu. G.: Space mappings with bounded distortion. - Transl. Math. Monogr. 73, Amer. Math. Soc., 1989.

[23] SARvas, J.: Ahlfors' trivial deformations and Liouville's theorem in $\mathbf{R}^{n}$. - In: Complex analysis, Joensuu 1978 (Proc. Colloq., Univ. Joensuu, Joensuu, 1978), Lecture Notes in Math. 747, Springer, Berlin, 1979, 343-348.

[24] SpIVAK, M.: A comprehensive introduction to differential geometry. Vol. III. Second edition. Publish or Perish, Inc., Wilmington, Del., 1979.

Received 25 April 2012 • Accepted 3 September 2012 